\title{
polymers
}

ISSN 2073-4360

www.mdpi.com/journal/polymers

Review

\section{Brillouin Scattering in Polymer Optical Fibers: Fundamental Properties and Potential Use in Sensors}

\section{Yosuke Mizuno * and Kentaro Nakamura}

Precision and Intelligence Laboratory, Tokyo Institute of Technology, 4259 Nagatsuta, Midori-ku, Yokohama 226-8503, Japan; E-Mail: knakamur@ sonic.pi.titech.ac.jp

* Author to whom correspondence should be addressed; E-Mail: ymizuno@ @onic.pi.titech.ac.jp; Tel.: +81-45-924-5052; Fax: +81-45-924-5091.

Received: 15 April 2011; in revised form: 9 May 2011 / Accepted: 25 May 2011 /

Published: 26 May 2011

\begin{abstract}
We review the fundamental properties of Brillouin scattering in a perfluorinated graded-index polymer optical fiber (PFGI-POF) with $120 \mu \mathrm{m}$ core diameter. The experiments were performed at $1.55 \mu \mathrm{m}$ telecommunication wavelength. The Brillouin frequency shift (BFS) and the Brillouin bandwidth were $2.83 \mathrm{GHz}$ and $105 \mathrm{MHz}$, respectively. The Brillouin gain coefficient was calculated to be $3.09 \times 10^{-11} \mathrm{~m} / \mathrm{W}$, which was comparable to that of fused silica fibers. The Brillouin threshold power of the $100 \mathrm{~m}$ POF was estimated to be as high as $24 \mathrm{~W}$, which can be, for practical applications, reduced by using POFs with smaller cores. These properties were compared with those of silica-based graded-index multi-mode fibers. We also investigated the BFS dependences on strain and temperature. They showed negative dependences with coefficients of $-121.8 \mathrm{MHz} / \%$ and $-4.09 \mathrm{MHz} / \mathrm{K}$, respectively, which are -0.2 and -3.5 times as large as those in silica fibers. These BFS dependences indicate that the Brillouin scattering in PFGI-POFs can be potentially applied to high-accuracy temperature sensing with reduced strain sensitivity.
\end{abstract}

Keywords: Brillouin scattering; polymer optical fiber; fiber-optic sensing; nonlinear optics 


\section{Introduction}

Compared to other standard glass fibers, polymer optical fibers (POFs) [1,2] are known to provide extremely easy and cost-effective connection; besides, they are flexible enough to endure over $40 \%$ strain [3]. Therefore, despite their higher loss than that of silica glass fibers, POFs have been utilized in medium-range communication applications such as home networks and automobiles [4], and in high-strain monitoring applications [3,5] as well. On the other hand, Brillouin scattering in optical fibers [6,7], which is one of the most significant nonlinear processes, has been widely studied. It has been applied to a number of useful devices and systems, such as optical amplifiers [7], lasers [7,8], optical comb generators [8], microwave signal processors [9], slow light generators [10], phase conjugators [11], tunable delay lines [12], and strain/temperature sensors [13-15]. Up to now, Brillouin scattering has been studied not merely for silica fibers but for some specialty fibers including tellurite glass fibers [16,17], $\mathrm{As}_{2} \mathrm{Se}_{3}$ chalcogenide fibers [18,19], bismuth-oxide highly-nonlinear fibers [20,21], and photonic crystal fibers [22,23]. However, Brillouin scattering in POFs has not yet been observed and reported, which will add a variety of advantages of POFs to the conventional application field of Brillouin scattering.

In this review, we summarize the fundamental properties of Brillouin scattering in a POF in the $1.55 \mu \mathrm{m}$ wavelength region [24]. The Brillouin frequency shift (BFS) of $2.83 \mathrm{GHz}$ and the Brillouin linewidth of $105 \mathrm{MHz}$ are measured. The calculated Brillouin gain coefficient $g_{\mathrm{B}}$ of $3.09 \times 10^{-11} \mathrm{~m} / \mathrm{W}$ is close to that of fused silica fibers. The Brillouin threshold power is estimated to be as high as $24 \mathrm{~W}$, which is in agreement with the theoretical predictions reported so far. These properties were compared with those of silica-based graded-index multi-mode fibers. For sensing applications, we also summarize the strain- and temperature-dependences of the BFS in the POF [25]. The BFS in the POF moves toward lower frequency with increasing strain and temperature with coefficients of $-121.8 \mathrm{MHz} / \%$ and $-4.09 \mathrm{MHz} / \mathrm{K}$, respectively. Compared to the coefficients of $+580 \mathrm{MHz} / \%$ and $+1.18 \mathrm{MHz} / \mathrm{K}$ in silica fibers, their absolute values are 0.2 and 3.5 times as large, respectively. These BFS dependences seem to be caused by the dependences of the Young's modulus on strain and temperature in the POF. We believe Brillouin scattering in POFs can be potentially used to implement high-accuracy temperature sensors with low strain sensitivity.

\section{Brillouin Scattering in Optical Fibers [7]}

When a light beam is injected into an optical fiber, it interacts with acoustic phonons, and generates backscattered light called Stokes light. This phenomenon is called spontaneous Brillouin scattering. Since the phonons decay exponentially, the backscattered Brillouin light spectrum, also known as Brillouin gain spectrum (BGS), takes the shape of Lorentzian function with the bandwidth of several tens of MHz. The frequency where the peak power is obtained in the BGS is down-shifted by several $\mathrm{GHz}$ from the incident light frequency, and the amount of this frequency shift is known as BFS. In optical fibers, the BFS $\nu_{\mathrm{B}}$ is given as [7]

$$
v_{B}=\frac{2 n v_{A}}{\lambda_{p}}=\frac{2 n}{\lambda_{p}} \sqrt{\frac{E}{\rho}}
$$


where $n$ is the refractive index, $v_{\mathrm{A}}$ the acoustic velocity in the fiber, $\lambda_{\mathrm{p}}$ the wavelength of the incident pump light, $E$ the Young's modulus, and $\rho$ the density. If tensile strain is applied or temperature is changed in a standard silica single-mode optical fiber (SMF), the BFS moves to higher frequency in proportion to the applied strain (+580 MHz/\%) [26] and the temperature change $(+1.18 \mathrm{MHz} / \mathrm{K})$ [27]. In some specialty fibers, such as tellurite glass fibers, it is known that the BFS moves to lower frequency with increasing applied strain $(-230 \mathrm{MHz} / \%)$ [17] and temperature $(-1.14 \mathrm{MHz} / \mathrm{K})$ [21]. In both cases, we can derive the strain amplitude and temperature change by measuring the BFS in the fiber.

\section{Experiments (I): Fundamental Properties}

In this section, we describe the experiments which reveal fundamental properties of Brillouin scattering in POFs, such as BFS, Brillouin linewidth, Brillouin gain coefficient, and Brillouin threshold power [24]. We also compare these properties with those of silica-based graded-index multimode fibers.

\subsection{Experimental Setup}

A standard POF composed of polymethyl methacrylate (PMMA) [1,2] is optimally designed for light transmission at $650 \mathrm{~nm}$, with a propagation loss of $\sim 200 \mathrm{~dB} / \mathrm{km}$. However, its loss at telecommunication wavelength is so high $\left(>>1 \times 10^{5} \mathrm{~dB} / \mathrm{km}\right)$ that the Brillouin signal cannot be detected. In the meantime, in order to observe Brillouin scattering in a PMMA-based POF at $650 \mathrm{~nm}$, we need to prepare all the necessary optical devices at this wavelength, which are quite difficult to prepare. Therefore, we used a 100-m perfluorinated graded-index POF (PFGI-POF) [28] instead of a PMMA-based POF. It has a numerical aperture (NA) of 0.185 , a core diameter of $120 \mu \mathrm{m}$, a core refractive index of 1.35 , and lower loss $(\sim 150 \mathrm{~dB} / \mathrm{km})$ even at $1.55 \mu \mathrm{m}$.

Figure 1 depicts the experimental setup for studying the Brillouin scattering properties in the PFGI-POF. For the BGS measurement with high resolution, we employed self-heterodyne detection [15]. All the optical paths except the POF itself were composed of silica SMFs. A distributed-feedback laser diode (DFB-LD) at 1,552 nm was employed as a light source, and its output was divided into two light beams with an optical coupler. One of the beams was, after passing a polarization controller (PC), directly used as the reference light of the heterodyne detection. The other beam was amplified with an erbium-doped fiber amplifier (EDFA) and injected into the POF as the pump light. Then, the optical beat signal between the backscattered Stokes light and the reference light was converted to an electrical signal with a photo-diode (PD). Finally, the signal was amplified by $23 \mathrm{~dB}$ with an electrical pre-amplifier, and monitored with an electrical spectrum analyzer (ESA).

We optically coupled the silica SMF and the POF using so-called butt coupling [29]. Since the core diameters are quite different ( $8 \mu \mathrm{m}$ of SMF vs. $120 \mu \mathrm{m}$ of POF), large optical loss is expected when light travels from the POF into the SMF. However, this loss contributes only to the attenuation of the Stokes light once generated in the POF, and it was measured to be approximately $12 \mathrm{~dB}$. On the other hand, when light travels from the SMF into the POF, the loss was less than $0.2 \mathrm{~dB}$, which is low enough to investigate the Brillouin scattering properties in the POF. 
Figure 1. Experimental setup for investigating the Brillouin scattering properties in the PFGI-POF. DFB-LD, distributed-feedback laser diode; EDFA, erbium-doped fiber amplifier; ESA, electrical spectrum analyzer; PC, polarization controller; PD, photo-diode.

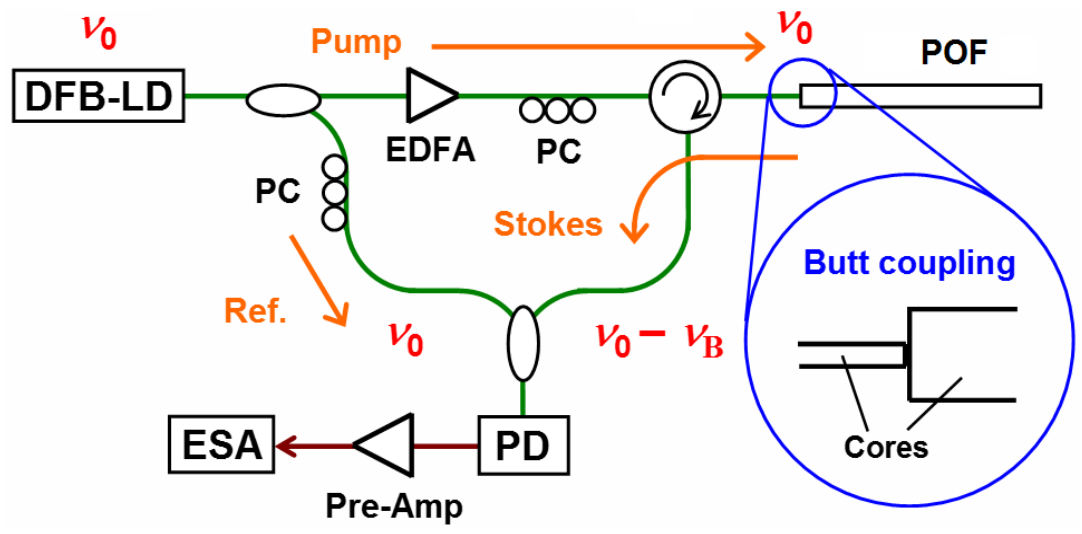

\subsection{Experimental Results}

The measured BGS when the $100 \mathrm{~m}$ PFGI-POF was pumped with $20 \mathrm{dBm}$ light is shown in Figure 2. The peak corresponding to the BFS was observed at $2.83 \mathrm{GHz}$, which is about 4 times lower than that of standard silica fibers. This allows the use of a PD and an ESA that are cheaper with lower bandwidth. The acoustic velocity $v_{\mathrm{A}}$ can be calculated using the BFS $v_{\mathrm{B}}$ as Equation (1). With $n$ of 1.35 and $\lambda_{\mathrm{p}}$ of $1,552 \mathrm{~nm}, v_{\mathrm{A}}$ in this POF was calculated to be $1,627 \mathrm{~m} / \mathrm{s}$, which is much lower than that of standard bulk PMMA, $\sim 2,700 \mathrm{~m} / \mathrm{s}$ [30]. By Lorentzian fitting, the $3 \mathrm{~dB}$ Brillouin linewidth $\Delta \nu_{\mathrm{B}}$ was measured to be $105 \mathrm{MHz}$, which is 3-5 times broader than that of silica fibers [31], resulting in deterioration of the sensitivity of time-domain sensors [13]. Here, we should bear in mind that the actual linewidth may be narrower due to the multimode nature of the PFGI-POF.

Figure 2. (a) BGS in the $100 \mathrm{~m}$ PFGI-POF when the pump power was $20 \mathrm{dBm}$.

(b) Magnified view around the BGS peak.

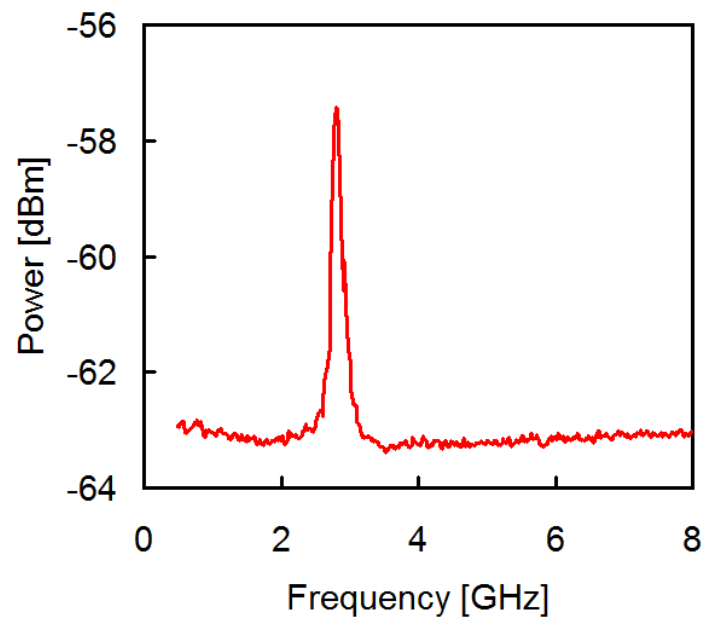

(a)

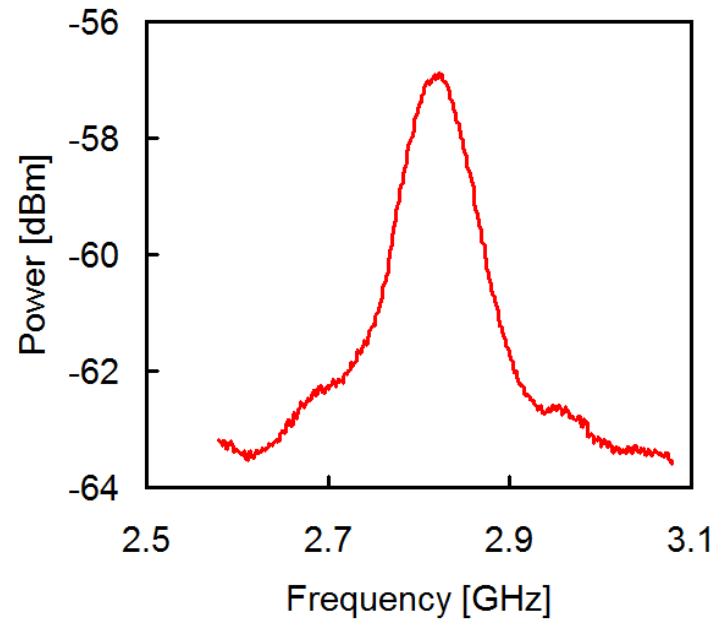

(b)

The dependence of the relative Stokes power on pump power is shown in Figure 3. The Stokes power is generally known to grow exponentially at the Brillouin threshold power $P_{\text {th }}$ and then reaches 
saturation, which indicates the transition from spontaneous to stimulated Brillouin scattering (SBS). Although rough estimation of $P_{\text {th }}$ is often performed using this kind of figure [7,16,18,31,32], the saturation of the Stokes power was not observed in Figure 3. Therefore, $P_{\text {th }}$ of this POF appears to be much higher than $30 \mathrm{dBm}$, i.e., $1 \mathrm{~W}$. The detailed estimation of $P_{\text {th }}$ will be given in Section 3.3.

Figure 3. Relative power of the Stokes light backscattered from $100 \mathrm{~m}$ PFGI-POF vs. pump power.

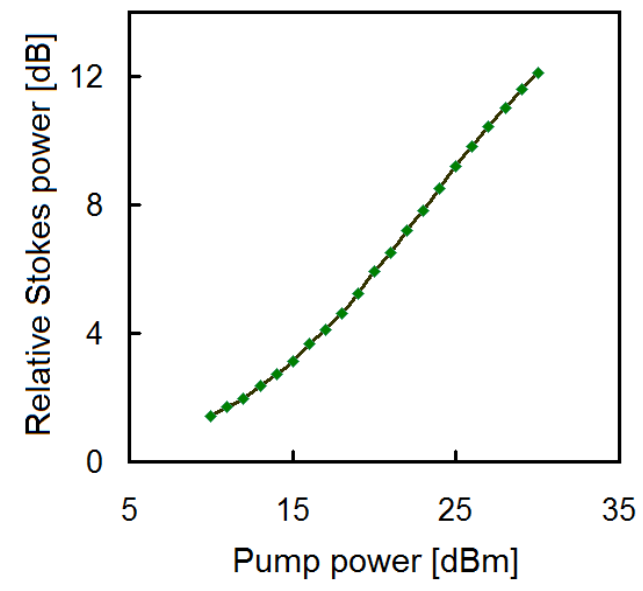

\subsection{Discussions}

First, we estimate the Brillouin gain coefficient $g_{\mathrm{B}}$. Using the acoustic velocity $v_{\mathrm{A}}$ and the Brillouin linewidth $\Delta v_{\mathrm{B}}, g_{\mathrm{B}}$ is given by [32]

$$
g_{B}=\frac{2 \pi n^{7} p_{12}{ }^{2}}{c \lambda_{p}^{2} \rho v_{A} \Delta v_{B}}
$$

where $p_{12}$ is the longitudinal elasto-optic coefficient, and $c$ the light velocity. Since the accurate values of $p_{12}$ and $\rho$ are not known for perfluorinated PMMA, we used the values of standard PMMA [33] in this calculation. Using the measured values of $v_{\mathrm{A}}=1,627 \mathrm{~m} / \mathrm{s}$ and $\Delta v_{\mathrm{B}}=105 \mathrm{MHz}$, along with $n=1.35, p_{12}=0.297, \lambda_{\mathrm{p}}=1,552 \mathrm{~nm}$, and $\rho=1,187.5 \mathrm{~kg} / \mathrm{m}^{3}, g_{\mathrm{B}}$ was calculated to be $3.09 \times 10^{-11} \mathrm{~m} / \mathrm{W}$, which is close to that of silica fibers $\left(3-5 \times 10^{-11} \mathrm{~m} / \mathrm{W}\right)$ [7]. Here, again, due to the multimode nature of the PFGI-POF, we should note that actual $g_{\mathrm{B}}$ may be larger than this value.

Then, we estimate the Brillouin threshold power $P_{\text {th }}$ of the PFGI-POF. An alternative way to calculate $g_{\mathrm{B}}$ is to use the following equation [34]:

$$
g_{B}=\frac{21 b A_{e f f}}{K P_{t h} L_{e f f}}
$$

where $A_{\text {eff }}$ is the effective cross-sectional area, and $L_{\text {eff }}$ is the effective length defined as

$$
L_{\text {eff }}=[1-\exp (-\alpha L)] / \alpha
$$

Here, $\alpha$ is the background loss and $L$ is the fiber length. For multimode fibers, a correction factor $b$ is needed [35], which can be treated as 2 when the NA is approximately 0.2 . $K$ is a constant that depends on the polarization properties of the fiber [32,36], which is 1 if the polarization is maintained and 0.667 otherwise. Then, using the values of $g_{\mathrm{B}}=3.09 \times 10^{-11} \mathrm{~m} / \mathrm{W}, b=2$ [35], $A_{\text {eff }}=209 \mu \mathrm{m}^{2}$ [37], $K=0.667, \alpha=0.056 / \mathrm{m}$, and $L=100 \mathrm{~m}, P_{\text {th }}$ can be calculated to be $24 \mathrm{~W}$. This is quite high but valid 
compared to the theoretical values of $\sim 10 \mathrm{~W}(L=300 \mathrm{~m})$ [37] or $\sim 100 \mathrm{~W}(L=100 \mathrm{~m})$ [38]. Since $P_{\text {th }}$ is in proportion to $b \cdot A_{\text {eff }}$ in Equation (3), $P_{\text {th }}$ can be decreased to a moderate power level by using POFs with smaller core diameters.

\subsection{Comparison with Silica-Based Graded-Index Multimode Fiber}

In order to compare the obtained properties of the PFGI-POF with those of a silica-based graded-index multimode fiber (GI-MMF), the Brillouin scattering in GI-MMF was experimentally characterized in the same manner. The setup was the same as in Figure 1 except that the electrical pre-amplifier was not used. The GI-MMF was $100 \mathrm{~m}$ in length, with a core diameter of $50 \mu \mathrm{m}$ and NA of 0.2. The connection loss of the butt coupling was less than $0.2 \mathrm{~dB}$ even when light travels from the GI-MMF into the SMF.

Figure 4 shows the measured BGS at the pump power of 15, 20, and $25 \mathrm{dBm}$, from which we obtained $10.43 \mathrm{GHz}$ as the BFS $v_{\mathrm{B}}$ and $47 \mathrm{MHz}$ as the Brillouin linewidth $\Delta v_{\mathrm{B}}$. Using Equation (2) with $n=1.46, p_{12}=0.286$ [39], $\lambda_{\mathrm{p}}=1,552 \mathrm{~nm}$, and $\rho=2,200 \mathrm{~kg} / \mathrm{m}^{3}$ [40], the Brillouin gain coefficient $g_{\text {B }}$ was calculated to be $1.63 \times 10^{-11} \mathrm{~m} / \mathrm{W}$, which was of the same order as that of silica SMFs $\left(3-5 \times 10^{-11} \mathrm{~m} / \mathrm{W}\right)[7]$.

Figure 4. BGS of the $100 \mathrm{~m}$ GI-MMF at the pump power of 15,20 , and $25 \mathrm{dBm}$.

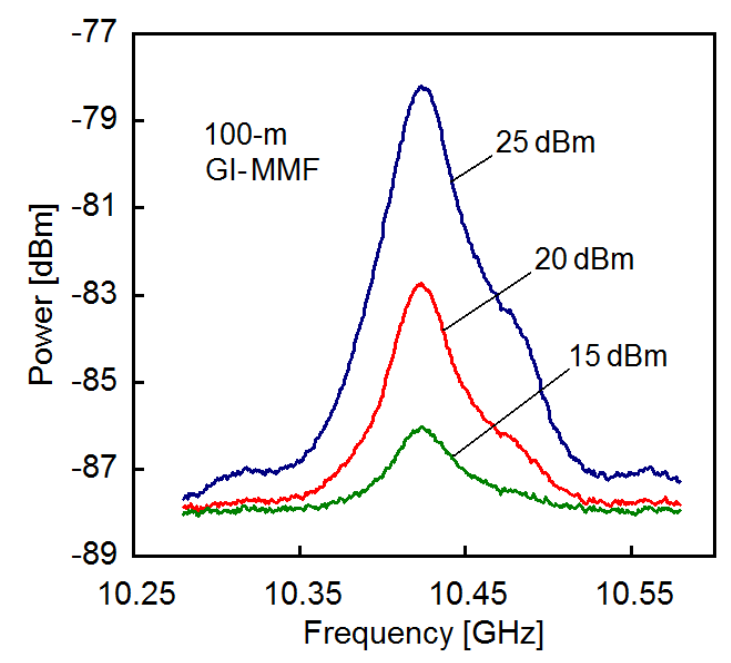

Figure 5 shows the dependence of the relative Stokes power on the pump power. The transition from spontaneous to stimulated Brillouin scattering was not observed. Using Equation (3) with $b=2$, $A_{\text {eff }}=131 \mu \mathrm{m}^{2}$ [41,42], $K=0.667, \alpha=0.000092 / \mathrm{m}$, and $L=100 \mathrm{~m}$, the Brillouin threshold power $P_{\text {th }}$ was calculated to be approximately $5 \mathrm{~W}$. This value is much higher than $\sim 0.3 \mathrm{~W}$, the typical $P_{\text {th }}$ of a $100 \mathrm{~m} \mathrm{SMF}[7,32]$. This is mostly due to the difference between the core diameters $(50 \mu \mathrm{m}$ of MMF vs. $\sim 10 \mu \mathrm{m}$ of SMF), since $P_{\text {th }}$ is in proportion to $A_{\text {eff }}$ as shown in Equation (3). On the other hand, the $5 \mathrm{~W}$ threshold of the GI-MMF is $\sim 30$ times lower than $P_{\text {th }}$ of a $100 \mathrm{~m}$ step-index (SI-) MMF, which is as high as $\sim 160 \mathrm{~W}$ according to Eichler et al's experiment [43]. We think the smaller mode dispersion of GI-MMFs is the cause of this difference, or in other words, $P_{\text {th }}$ of GI-MMFs is lower probably because the fundamental mode is mainly excited, while multiple modes are excited in SI-MMFs. 
Figure 5. Relative Stokes power as a function of pump power in the $100 \mathrm{~m}$ GI-MMF.

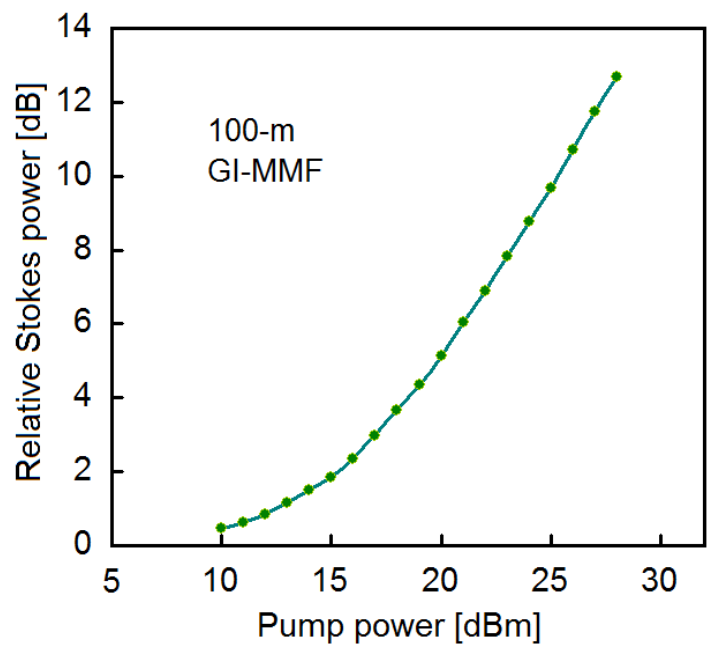

In Table 1, the properties of the two fibers are summarized. We believe this information will be a useful guideline for developing POF-based systems in future.

Table 1. Comparison of the material properties between the PFGI-POF and the GI-MMF used in this experiment: $L$, fiber length; $N A$, numerical aperture; $d$, core diameter; $n$, approximate refractive index of the core; $A_{\text {eff }}$, effective cross-sectional area; $v_{\mathrm{B}}$, Brillouin frequency shift; $v_{\mathrm{A}}$, acoustic velocity; $\Delta v_{\mathrm{B}}$, Brillouin linewidth; $g_{\mathrm{B}}$, Brillouin gain coefficient; $P_{\text {th }}$, Brillouin threshold power.

\begin{tabular}{|c|c|c|c|c|c|c|c|c|c|c|}
\hline & $\boldsymbol{L}[\mathbf{m}]$ & $\boldsymbol{N A}$ & $\boldsymbol{d}[\mathbf{m m}]$ & $\boldsymbol{n}$ & $\boldsymbol{A}_{\text {eff }}\left[\mathbf{m m}^{2}\right]$ & $\boldsymbol{\nu}_{\mathrm{B}}[\mathbf{G H z}]$ & $\boldsymbol{v}_{\mathrm{A}}[\mathbf{m} / \mathbf{s}]$ & $\boldsymbol{\Delta} \boldsymbol{\nu}_{\mathrm{B}}[\mathbf{M H z}]$ & $\boldsymbol{g}_{\mathrm{B}}\left[\times \mathbf{1 0}^{-\mathbf{1 1}} \mathbf{m} / \mathbf{W}\right]$ & $\boldsymbol{P}_{\text {th }}[\mathbf{W}]$ \\
\hline PFGI-POF & 100 & 0.185 & 120 & 1.35 & 209 & 2.83 & 1,627 & 105 & 3.09 & 24 \\
\hline GI-MMF & 100 & 0.2 & 50 & 1.46 & 131 & 10.43 & 5,544 & 47 & 1.63 & 5 \\
\hline
\end{tabular}

\section{Experiments (II): Potential Use in Sensors}

In this section, we describe the experiments for investigating the strain- and temperature-dependences of the BFS in the PFGI-POF, and clarify that Brillouin scattering in PFGI-POFs can be potentially utilized to develop high-accuracy temperature sensors with reduced strain sensitivity [25]. We also show that these BFS dependences are probably caused by the dependences of the Young's modulus on strain and temperature in the PFGI-POF.

\subsection{Experimental Setup}

We used a short PFGI-POF $(5 \mathrm{~m})$ with the same physical properties as that used in the previous experiments. The experimental setup for investigating the BFS dependence on strain and temperature in the PFGI-POF is basically the same as that shown in Figure 1. The Brillouin signal generated in the $1 \mathrm{~m} \mathrm{SMF}$ between the circulator and the PFGI-POF is included in the Stokes light, but it has no influence on the BGS measurement, because the BFS in the SMF is typically $11 \mathrm{GHz}$, about 4 times higher than that in the PFGI-POF. The whole length of the PFGI-POF was fixed on a translation stage using epoxy glue, to which different strains were applied. Temperature was adjusted with a heater along the whole length of the PFGI-POF. 


\subsection{Experimental Results}

Figure 6(a) shows the strain-dependence of the BGS in the PFGI-POF. The pump power was $19 \mathrm{dBm}$, and strains of $0.2,0.4,0.6,0.8$ and $1.0 \%$ were applied. As the applied strain increased, the BGS shifted toward lower frequency. Figure 6(b) shows the strain-dependence of the BFS. The slope was almost linear, and its coefficient was $-121.8 \mathrm{MHz} / \%$. While the negative sign is the same as in tellurite glass fibers [17], the absolute value was approximately one fifth of that of a standard silica SMF (+580 MHz/\%) [26].

Figure 6. (a) BGS dependence on strain in the PFGI-POF. (b) BFS as a function of applied strain.

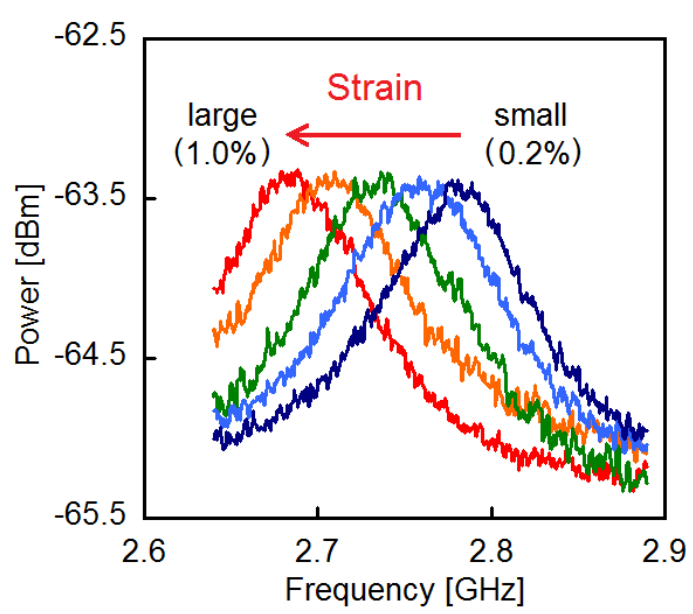

(a)

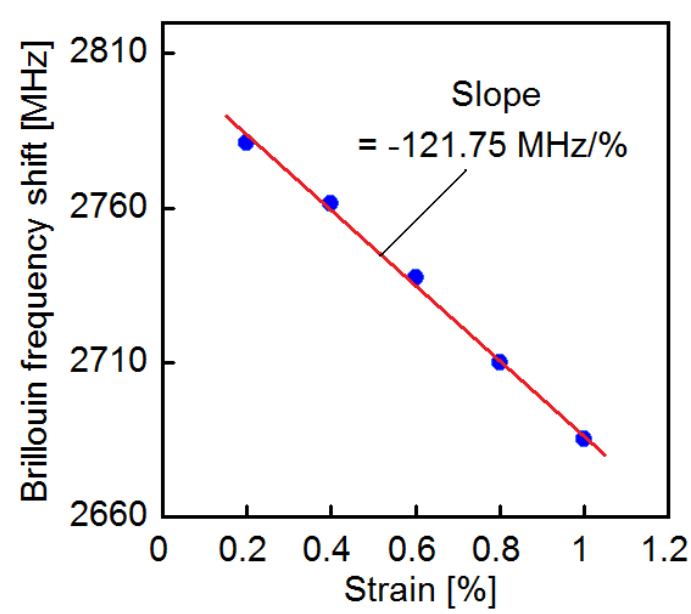

(b)

Then, Figure 7(a) shows the temperature-dependence of the BGS in the PFGI-POF. The pump power was $23 \mathrm{dBm}$, and the temperature was controlled from $30{ }^{\circ} \mathrm{C}$ up to $80{ }^{\circ} \mathrm{C}$ with a step of $10{ }^{\circ} \mathrm{C}$. As temperature increased, the BGS also shifted toward lower frequency. The Stokes power at high temperature over $40^{\circ} \mathrm{C}$ was lower than that at $30^{\circ} \mathrm{C}$ by about $0.7 \mathrm{~dB}$ probably because of the nonuniform temperature distribution on the heater. Figure 7(b) shows the temperature-dependence of the BFS, and its coefficient was $-4.09 \mathrm{MHz} / \mathrm{K}$. Though the negative sign is also the same as in tellurite glass fibers [21], the absolute value was about 3.5 times as large as that of an SMF (+1.18 MHz/K) [27].

Figure 7. (a) BGS dependence on temperature in the PFGI-POF. (b) BFS as a function of temperature.

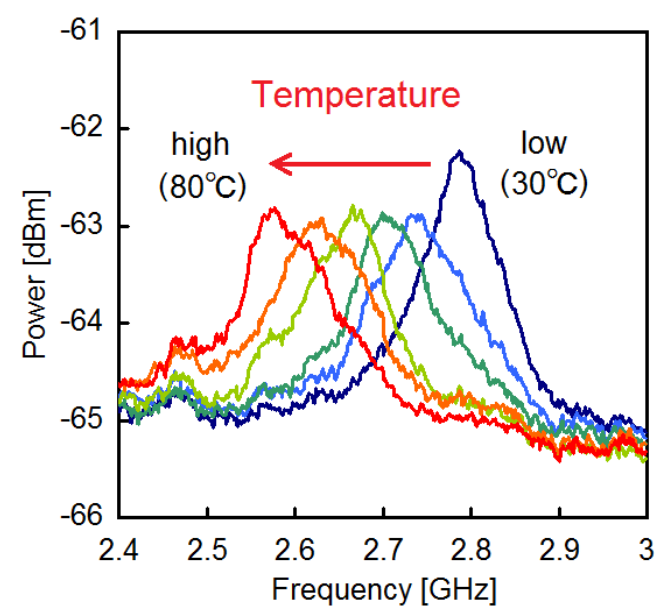

(a)

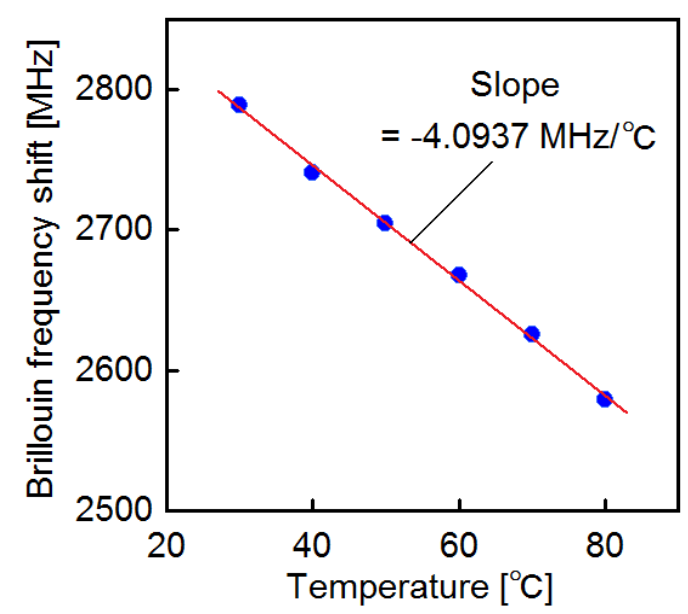

(b) 
The larger temperature coefficient leads to accuracy enhancement of the temperature measurement. On the other hand, the smaller strain coefficient means that PFGI-POF-based Brillouin sensors are less susceptible to the applied strain. Therefore, the Brillouin scattering in the PFGI-POF can be potentially utilized to implement high-accuracy temperature sensors with low strain sensitivity.

\subsection{Discussions}

The origins of the BFS dependences on strain and temperature in the PFGI-POF are discussed. The strain coefficient of the normalized BFS is given, by differentiating Equation (1) with respect to strain, as:

$$
\frac{1}{v_{B}} \frac{\partial v_{B}}{\partial \varepsilon}=\frac{1}{n} \frac{\partial n}{\partial \varepsilon}+\frac{1}{2 E} \frac{\partial E}{\partial \varepsilon}+\left(-\frac{1}{2 \rho} \frac{\partial \rho}{\partial \varepsilon}\right)
$$

here the following two equations hold true [26]:

$$
\begin{gathered}
\frac{1}{n} \frac{\partial n}{\partial \varepsilon}=-n^{2} \frac{p_{12}-\kappa\left(p_{11}+p_{12}\right)}{2} \\
-\frac{1}{\rho} \frac{\partial \rho}{\partial \varepsilon}=\frac{1-2 \kappa}{2}
\end{gathered}
$$

where $p_{11}$ and $p_{12}$ are the elasto-optic coefficients, and $\kappa$ is the Poisson's ratio. As their values in PFGI-POF are unknown, we used the values for bulk PMMA: $p_{11}=0.3$ [44], $p_{12}=0.297$ [44], and $\kappa=0.34$ [45]. Then the first and the third terms in Equation (5) were calculated to be -0.0857 and +0.16 , respectively. Though the second term is reported to drastically vary depending both on the method to apply strain and on the fabrication quality of the fiber, we used -5.75 as the second term, which is the value reported in [46] for a standard PMMA-based POF. Compared to its absolute value, the first and the third terms are negligibly small. Then the theoretical strain coefficient was calculated to be $-160.6 \mathrm{MHz} / \%$. Considering that each term in Equation (5) was estimated using the values of PMMA, this value is in moderate agreement with the experimental value of $-121.8 \mathrm{MHz} / \%$. Thus, the strain-dependence of the BFS appears to originate from the dependence of the Young's modulus on strain in the PFGI-POF.

Next, we discuss the BFS dependence on temperature in the same manner. The temperature coefficient of the normalized BFS is expressed as

$$
\frac{1}{v_{B}} \frac{\partial v_{B}}{\partial T}=\frac{1}{n} \frac{\partial n}{\partial T}+\frac{1}{2 E} \frac{\partial E}{\partial T}+\left(-\frac{1}{2 \rho} \frac{\partial \rho}{\partial T}\right)
$$

the first term can be calculated to be -0.0000889 referring to the value reported for a standard PMMA-based POF [33]. In order to estimate the second and the third terms, we plotted the Young's modulus and the density of bulk PMMA at various temperatures using the data in [47], as shown in Figure $8(\mathrm{a}, \mathrm{b})$. Using their slopes $\left(-0.0295 \mathrm{GPa} / \mathrm{K}\right.$ and $\left.-0.409 \mathrm{~kg} / \mathrm{m}^{3} / \mathrm{K}\right)$, along with $E \sim 6 \mathrm{GPa}$ and $\rho=1,187.5 \mathrm{~kg} / \mathrm{m}^{3}$, the second and the third terms were calculated to be -0.00246 and +0.000172 , respectively. Then the theoretical temperature coefficient was calculated to be $-6.72 \mathrm{MHz} / \mathrm{K}$, which is in rough agreement with the experimental value of $-4.09 \mathrm{MHz} / \mathrm{K}$. Thus, the temperature-dependence 
of the BFS also seems to originate from the large negative dependence of the Young's modulus on temperature in the PFGI-POF.

Figure 8. (a) Young's modulus of bulk PMMA vs. temperature. (b) Density of bulk PMMA vs. temperature (plotted using the data reported in [47]).

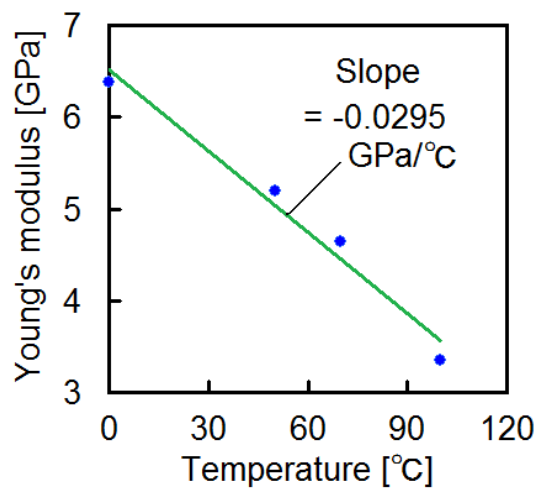

(a)

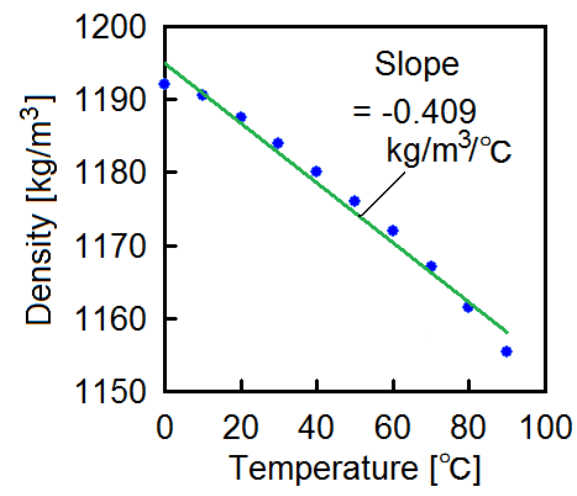

(b)

\section{Conclusions}

We made a review on the Brillouin scattering properties in the PFGI-POF at $1.55 \mu \mathrm{m}$ wavelength. The BFS and the Brillouin linewidth were $2.83 \mathrm{GHz}$ and $105 \mathrm{MHz}$, respectively. Using these values, the Brillouin gain coefficient was calculated to be $3.09 \times 10^{-11} \mathrm{~m} / \mathrm{W}$, which is almost the same as that of standard silica SMFs and higher than that of silica GI-MMFs. Here, we should bear in mind the actual Brillouin linewidth and the actual Brillouin gain coefficient may be narrower and higher, respectively, than these values due to the multimode nature of the PFGI-POF. This fact indicates that, as Brillouin scattering in silica fibers has wide application fields, Brillouin scattering in POFs can also be utilized to develop a number of medium-range practical devices and systems with their low cost, ease of installation, high flexibility, and high safety.

We also summarized the BFS dependences on strain and temperature in a $5 \mathrm{~m}$ PFGI-POF. They showed negative dependences with coefficients of $-121.8 \mathrm{MHz} / \%$ and $-4.09 \mathrm{MHz} / \mathrm{K}$, respectively, which are -0.2 and -3.5 times as large as those in silica fibers. These BFS dependences were found to originate from the dependences of the Young's modulus on strain and temperature. Thus, Brillouin scattering in PFGI-POFs has a big potential for high-accuracy temperature sensing with reduced strain sensitivity.

For the implementation of such sensing systems, the most important problem to be solved is the extremely low power of the Stokes light, which is associated with the Brillouin threshold power of as high as $24 \mathrm{~W}$. In other words, low signal-to-noise ratio (SNR) is the major disadvantage of currently feasible Brillouin-based distributed POF sensors as compared to Rayleigh-based POF sensors [5]. As discussed in Section 3.3, one way to enhance the Brillouin signal is to employ POFs with smaller core diameters. Another method is to observe SBS, not spontaneous scattering, based on so-called pump-probe technique [48]. We expect further research will extend the potential of Brillouin scattering in POFs not only for novel sensors but also for other useful devices and systems. 


\section{Acknowledgements}

We are indebted to Nishiyama and Uenohara of Tokyo Institute of Technology, Japan, for lending us their high-power EDFAs. Y. Mizuno is grateful to the Research Fellowships for Young Scientists from the Japan Society for the Promotion of Science (JSPS).

\section{References}

1. Kuzyk, M.G. Polymer Fiber Optics: Materials, Physics, and Applications, 1st ed.; CRC Press: Boca Raton, FL, USA, 2006.

2. Koike, Y.; Ishigure, T.; Nihei, E. High-bandwidth graded-index polymer optical fiber. J. Lightw. Technol. 1995, 13, 1475-1489.

3. Husdi, I.R.; Nakamura, K.; Ueha, S. Sensing characteristics of plastic optical fibres measured by optical time-domain reflectometry. Meas. Sci. Technol. 2004, 15, 1553-1559.

4. Mollers, I.; Jager, D.; Gaudino, R.; Nocivelli, A.; Kragl, H.; Ziemann, O.; Weber, N.; Koonen, T.; Lezzi, C.; Bluschke, A.; Randel, S. Plastic optical fiber technology for reliable home networking-overview and results of the EU project POF-ALL. IEEE Commun. Mag. 2009, 47, 58-68.

5. Liehr, S.; Lenke, P.; Wendt, M.; Krebber, K.; Seeger, M.; Thiele, E.; Metschies, H.; Gebreselassie, B.; Munich, J.C. Polymer optical fiber sensors for distributed strain measurement and application in structural health monitoring. IEEE Sens. J. 2009, 9, 1330-1338.

6. Ippen, E.P.; Stolen, R.H. Stimulated Brillouin scattering in optical fibers. Appl. Phys. Lett. 1972, 21, 539-541.

7. Agrawal, G.P. Nonlinear Fiber Optics, 4th ed.; Academic Press: San Diego, CA, USA, 1995; pp. 329-367.

8. Cowle, G.J.; Yu, D.; Chieng, Y.T. Brillouin/erbium fiber lasers. J. Lightw. Technol. 1997, 15, 1198-1204.

9. Norcia, S.; Tonda-Goldstein, S.; Dolfi, D.; Huignard, J.P. Efficient single-mode Brillouin fiber laser for low-noise optical carrier reduction of microwave signals. Opt. Lett. 2003, 28, 1888-1890.

10. Song, K.Y.; Herraez, M.G.; Thevenaz, L. Observation of pulse delaying and advancement in optical fibers using stimulated Brillouin scattering. Opt. Express 2005, 13, 82-88.

11. Kuzin, E.A.; Petrov, M.P.; Davydenko, B.E. Phase conjugation in an optical fibre. Opt. Quantum Electon. 1985, 17, 393-397.

12. Zou, W.; He, Z.; Hotate, K. Tunable fiber-optic delay line based on stimulated Brillouin scattering. Appl. Phys. Express 2010, 3, 012501.

13. Horiguchi, T.; Tateda, M. BOTDA-nondestructive measurement of single-mode optical fiber attenuation characteristics using Brillouin interaction: Theory. J. Lightw. Technol. 1989, 7, 1170-1176.

14. Hotate, K.; Hasegawa, T. Measurement of Brillouin gain spectrum distribution along an optical fiber using a correlation-based technique-proposal, experiment and simulation. IEICE Trans. Electron. 2000, E83-C, 405-412.

15. Mizuno, Y.; Zou, W.; He, Z.; Hotate, K. Proposal of Brillouin optical correlation-domain reflectometry (BOCDR). Opt. Express 2008, 16, 12148-12153. 
16. Abedin, K.S. Stimulated Brillouin scattering in single-mode tellurite glass fiber. Opt. Express 2006, 14, 11766-11772.

17. Mizuno, Y.; He, Z.; Hotate, K. Distributed strain measurement using a tellurite glass fiber with Brillouin optical correlation-domain reflectometry. Opt. Commun. 2010, 283, 2438-2441.

18. Abedin, K.S. Observation of strong stimulated Brillouin scattering in single-mode $\mathrm{As}_{2} \mathrm{Se}_{3}$ chalcogenide fiber. Opt. Express 2005, 13, 10266-10271.

19. Song, K.Y.; Abedin, K.S.; Hotate, K.; Herraez, M.G.; Thevenaz, L. Highly efficient Brillouin slow and fast light using $\mathrm{As}_{2} \mathrm{Se}_{3}$ chalcogenide fiber. Opt. Express 2006, 14, 5860-5865.

20. Lee, J.H.; Tanemura, T.; Kikuchi, K.; Nagashima, T.; Hasegawa, T.; Ohara, S.; Sugimoto, N. Experimental comparison of a Kerr nonlinearity figure of merit including the stimulated Brillouin scattering threshold for state-of-the-art nonlinear optical fibers. Opt. Lett. 2005, 30, 1698-1700.

21. Mizuno, Y.; He, Z.; Hotate, K. Dependence of the Brillouin frequency shift on temperature in a tellurite glass fiber and a bismuth-oxide highly-nonlinear fiber. Appl. Phys. Express 2009, 2, 112402.

22. Lee, J.H.; Yusoff, Z.; Belardi, W.; Ibsen, M.; Monro, T.M.; Richardson, D.J. Investigation of Brillouin effects in small-core holey optical fiber: lasing and scattering. Opt. Lett. 2002, 27, 927-929.

23. Beugnot, J.C.; Sylvestre, T.; Alasia, D.; Maillotte, H.; Laude, V.; Monteville, A.; Provino, L.; Traynor, N.; Mafang, S.F.; Thevenaz, L. Complete experimental characterization of stimulated Brillouin scattering in photonic crystal fiber. Opt. Express 2007, 15, 15517-15522.

24. Mizuno, Y.; Nakamura, K. Experimental study of Brillouin scattering in perfluorinated polymer optical fiber at telecommunication wavelength. Appl. Phys. Lett. 2010, 97, 021103.

25. Mizuno, Y.; Nakamura, K. Potential of Brillouin scattering in polymer optical fiber for strain-insensitive high-accuracy temperature sensing. Opt. Lett. 2010, 35, 3985-3987.

26. Horiguchi, T.; Kurashima, T.; Tateda, M. Tensile strain dependence of Brillouin frequency shift in silica optical fibers. IEEE Photon. Technol. Lett. 1989, 1, 107-109.

27. Kurashima, T.; Horiguchi, T.; Tateda, M. Thermal effects on the Brillouin frequency shift in jacketed optical silica fibers. Appl. Opt. 1990, 29, 2219-2222.

28. Ishigure, T.; Koike, Y.; Fleming, J.W. Optimum index profile of the perfluorinated polymer-based GI polymer optical fiber and its dispersion properties. J. Lightw. Technol. 2000, 18, 178-184.

29. Sidorin, Y.; Howe, D. Laser-diode wavelength tuning based on butt coupling into an optical fiber. Opt. Lett. 1997, 22, 802-804.

30. Hattori, K.; Matsuoka, T.; Sakai, K.; Takagi, K. Light beating spectroscopy of Brillouin scattering in solid polymer. Jpn. J. Appl. Phys. 1994, 33, 3217-3219.

31. Yeniay, A.; Delavaux, J.M.; Toulouse, J. Spontaneous and stimulated Brillouin scattering gain spectra in optical fibers. J. Lightwave Technol. 2002, 20, 1425-1432.

32. Cotter, D. Observation of stimulated Brillouin scattering in low-loss silica fibre at $1.3 \mu \mathrm{m}$. Electron. Lett. 1982, 18, 495-496.

33. Silva-Lopez, M.; Fender, A.; MacPherson, W.N.; Barton, J.S.; Jones, J.D.C.; Zhao, D.; Dobb, H.; Webb, D.J.; Zhang, L.; Bennion, I. Strain and temperature sensitivity of a single-mode polymer optical fiber. Opt. Lett. 2005, 30, 3129-3131. 
34. Smith, R.G. Optical power handling capacity of low loss optical fibers as determined by stimulated Raman and Brillouin scattering, Appl. Opt. 1972, 11, 2489-2494.

35. Tei, K.; Tsuruoka, Y.; Uchiyama, T.; Fujioka, T. Critical power of stimulated Brillouin scattering in multimode optical fibers. Jpn. J. Appl. Phys. 2001, 40, 3191-3194.

36. Zadok, A.; Zilka, E.; Eyal, A.; Thevenaz, L.; Tur, M. Vector analysis of stimulated Brillouin scattering amplification in standard single-mode fibers. Opt. Express 2008, 16, 21692-21707.

37. Dossou, M.; Szriftgiser, P.; Goffin, A. Theoretical study of stimulated Brillouin scattering (SBS) in polymer optical fibres. Proc. Symp. IEEE/LEOS Benelux Chapt. 2008, 13, 175-178.

38. Lenke, P.; Nother, N. Stimulated Brillouin scattering in graded index multimode optical fiber by excitation of the fundamental mode only. Proc. SPIE 2007, 6582, 658213.

39. Faris, G.W.; Jusinski, L.E.; Hickman, A.P. High-resolution stimulated Brillouin gain spectroscopy in glasses and crystals. J. Opt. Soc. Am. B 1993, 10, 587-599.

40. Uchida, N.; Niizeki, N. Acousto-optic deflection materials and techniques. Proc. IEEE 1973, 61, 1073-1092.

41. Morgan, J.D.S. Backward Amplification and Beam Cleanup of a Raman Fiber Laser Oscillator Using a Multi-Mode Graded Index fiber AMPLIFIER. M.S. Thesis, Air Force Institute of Technology, Air University, Wright-Patterson AFB, OH, USA, 2006.

42. Marcuse, D. Loss analysis of single-mode fiber splices. Bell Syst. Technol. J. 1977, 56, 703-718.

43. Eichler, H.J.; Mocofanescu, A.; Riesbeck, T.; Risse, E.; Bedau, D. Stimulated Brillouin scattering in multimode fibers for optical phase conjugation. Opt. Commun. 2002, 208, 427-431.

44. Waxler, R.M.; Horowitz, D.; Feldman, A. Optical and physical parameters of Plexiglas 55 and Lexan. Appl. Opt. 1979, 18, 101-104.

45. Raftopoulos, D.D.; Karapanos, D.; Theocaris, P.S. Static and dynamic mechanical and optical behaviour of high polymers. J. Phys. D 1976, 9, 869-877.

46. Kiesel, S.; Peters, K.; Hassan, T.; Kowalsky, M. Behaviour of intrinsic polymer optical fibre sensor for large-strain applications. Meas. Sci. Technol. 2007, 18, 3144-3154.

47. Saneyoshi, J.; Kikuchi, Y.; Nomoto, O. Handbook of Ultrasonic Technology, 1st ed.; Nikkan Kogyo: Tokyo, Japan, 1978; Chapter 5.

48. Shibata, N.; Azuma, Y.; Horiguchi, T.; Tateda, M. Identification of longitudinal acoustic modes guided in the core region of a single-mode optical fiber by Brillouin gain spectra measurements. Opt. Lett. 1988, 13, 595-597.

(C) 2011 by the authors; licensee MDPI, Basel, Switzerland. This article is an open access article distributed under the terms and conditions of the Creative Commons Attribution license (http://creativecommons.org/licenses/by/3.0/). 